\title{
Research on Video Segmentation and Motion Detection Based on Lattice Pohl Seidman Method
}

\author{
Xiao-Dong $\mathrm{ZHU}^{1}$ and Jing WANG ${ }^{2}$ \\ ${ }^{1}$ Ningbo City College of Vocation Technology, Ningbo, China \\ 2 Zhejiang International Studies University, Hangzhou, China \\ 1zxdningbo@126.com
}

\begin{abstract}
Keywords: Video analysis, image segmentation, Lattice Lattice Boltzmann Method, optical flow field, motion estimation, threshold setting
\end{abstract}

\begin{abstract}
This paper mainly studies the application of the lattice Pohl Seidman method in the optical flow field computer. A lattice Pohl Seidman equation is established for the optical flow field in a sequence of consecutive image frames using the lattice Pohl Seidman method. And the corresponding constraint conditions are set up. By using the lattice D2Q9 equation of the Pohl Seidman equation in the form of a lattice equation, the computational equation is used to calculate the optical flow field. The image can be segmented accurately by the iterative results, and the local motion estimation can be realized. The simulation results show that the detection results are accurate. It simulation results show that the detection results are accurate. Experimental results show that the proposed method is more than one order of magnitude faster than Laplasse filtering. It has important significance for video coding.
\end{abstract}

\section{Introduction}

Lattice Pohl Seidman (Boltzmann) method has been developed rapidly in the past more than and 20 years with the solution of various dynamic fluid mechanics problems. This method is a new theory and method developed from lattice gas automata theory [1,2]. This method can be used directly as the Boltzmann equation. It can solve the problem of computational fluid dynamics, such as solving the Stokes (Navier-Stokes (NS)) equation, and obtaining the velocity U and pressure P[3]. B.Jawerth et al. First proposed the application of the lattice Pohl Seidman model to the inhomogeneous image processing [4]. The goal of this study is to establish a formal lattice Pohl Seidman equation for the optical flow field in image sequences. The approximate condition of the optical flow equation is modified by the approximate treatment of the lattice Pohl Seidman formula, and the problem of the edge detection of the approximate processing is better solved. Meanwhile, the problem of image sequence segmentation can be effectively processed [5].

\section{Lattice Pohl Seidman Equation of Optical Flow Field}

How to search the moving objects in image sequences is the most important step for local motion estimation. In the process of continuous video frame image motion estimation, the optical flow field is divided into small blocks which are connected with each other. Thus, the motion vector of the moving object can be estimated according to the vector field of each block[6]. Most of the current optical flow equations are based on the improved Horn-Shunk's backward smoothing method and the Lucas-Kanad's forward smoothing method. The academic session also includes other improvements. The core idea of these methods is to add a variety of constraints in the derivation of the optical flow equation based on the image sequence.

The disadvantage of these algorithms is that the computational complexity is too high. The emphasis of this study is to modify the constraint equation of optical flow field. Motion vector estimation is carried out by using the formal lattice Pohl Seidman equation, and the moving boundary can be detected by analyzing the continuous motion vector field [7].

For any continuous video, assuming that $F\left(x_{1}, y_{1}, t\right)$ is a frame in the video sequence at the $\mathrm{t}$ moment, (x1, Y1) represents the Descartes coordinates of the pixels in the current frame image. The 
interval between consecutive image frames is $\Delta t . \mathbf{v}=\left(x_{1}, y_{1}\right)$ is the representation of pixel motion vector function. $F(\mathbf{v}, t)$ is a two - differentiable continuous function. At the same time, the result of $F(\mathbf{v}+\mu \Delta t, t+\Delta t)$ is expanded by using Taylor formula as:

$$
F(\mathbf{v}+\mu \Delta t, t+\Delta t)=F(\mathbf{v}, t)+\Delta t\left(\mu \cdot\left(\frac{\partial}{\partial x_{1}}, \frac{\partial}{\partial y_{1}}\right)+\frac{\partial}{\partial t}\right) F(\mathbf{v}, t)+\frac{\Delta t^{2}}{2 !}\left(\mu \cdot\left(\frac{\partial}{\partial x_{1}}, \frac{\partial}{\partial y_{1}}\right)+\frac{\partial}{\partial t}\right)^{2} F(\mathbf{v}, t)+O\left(\Delta t^{3}\right)
$$

$\mu$ is a very small two-dimensional vector, representing a pre estimated motion vector, as the initial value of the iterative calculation. Set $\mathbf{v}(t)$ to indicate the position of a pixel at a time. $\mathbf{v}(t+\Delta t)$ indicates that the pixel is moved to a new location after the $\Delta t$ interval, and the F function represents the coordinates of the position change relation can be expressed as:

$$
F(\mathbf{v}(t+\Delta t), t+\Delta t)=F(\mathbf{v}, t)+\Delta t\left(\frac{\mathrm{d} \mathbf{v}}{\mathrm{d} t} \cdot\left(\frac{\partial}{\partial x_{1}}, \frac{\partial}{\partial y_{1}}\right)+\frac{\partial}{\partial t}\right) F(\mathbf{v}, t)+\frac{\Delta t^{2}}{2 !}\left(\frac{\mathrm{d} \mathbf{v}}{\mathrm{d} t} \cdot\left(\frac{\partial}{\partial x_{1}}, \frac{\partial}{\partial y_{1}}\right)+\frac{\partial}{\partial t}\right)^{2} F(\mathbf{v}, t)+O\left(\Delta t^{3}\right)
$$

The motion estimation of pixels is to obtain the motion vector $\mathrm{d} \mathbf{v} / \mathrm{d} t$ of the pixel, and construct the following functions:

$$
\begin{aligned}
& \omega(F, \mu, t, \Delta t)=(F(\mathbf{v}+\mu \Delta t, t+\Delta t)-F(\mathbf{v}, t)) / \Delta t-F(\mathbf{v}, t) / \Delta t-\frac{\Delta t^{2}}{2 !}\left(\mu \cdot\left(\frac{\partial}{\partial x_{1}}, \frac{\partial}{\partial y_{1}}\right)+\frac{\partial}{\partial t}\right)^{2} F(\mathbf{v}, t)+O\left(\Delta t^{3}\right) \\
& \omega^{\prime}\left(F, \frac{\mathrm{d} \mathbf{v}}{\mathrm{d} t}, t, \Delta t\right)=\left(F\left(\mathbf{v}+\frac{\mathrm{d} \mathbf{v}}{\mathrm{d} t} \Delta t, t+\Delta t\right)-F(\mathbf{v}, t)\right) / \Delta t-F(\mathbf{v}, t) / \Delta t-\frac{\Delta t^{2}}{2 !}\left(\frac{\mathrm{d} \mathbf{v}}{\mathrm{d} t} \cdot\left(\frac{\partial}{\partial x_{1}}, \frac{\partial}{\partial y_{1}}\right)+\frac{\partial}{\partial t}\right)^{2} F(\mathbf{v}, t)+O\left(\Delta t^{3}\right)
\end{aligned}
$$

The following equations can be obtained by collating and simplifying equations (1) and (2):

$$
\begin{gathered}
\omega(F, \mu, t, \Delta t)=\left(\mu \cdot\left(\frac{\partial}{\partial x_{1}}, \frac{\partial}{\partial y_{1}}\right)+\frac{\partial}{\partial t}\right) F(\mathbf{v}, t) \\
\omega^{\prime}\left(F, \frac{\mathrm{d} \mathbf{v}}{\mathrm{d} t}, t, \Delta t\right)=\left(\frac{\mathrm{d} \mathbf{v}}{\mathrm{d} t} \cdot\left(\frac{\partial}{\partial x_{1}}, \frac{\partial}{\partial y_{1}}\right)+\frac{\partial}{\partial t}\right) F(\mathbf{v}, t)
\end{gathered}
$$

Since the parameters used are the estimated vector and the actual vector, the $\omega$ function is different from the $\omega^{\prime}$ function. In the vector field of moving pixels, the value of the function cannot be guaranteed to be zero, which is because the law of conservation of light is not always guaranteed. These functions are often only approximately equal to zero, the formula (6) is the general optical flow field conservation formula, also is the formalization optical flow field lattice Pohl Seidman equation, the formula (5) is the auxiliary function. The general formula for the conservation of the optical flow field is used to describe the motion, in which the parameter $\mathrm{d} \mathbf{v} / \mathrm{d} t$ cannot be obtained directly. As the $\omega$ function is the same as the $\omega^{\prime}$ function, the parameters should be the same. In order to simplify the calculation, the approximate method is used to simplify the two equations. Through the formula (5) to estimate the motion parameters, the prediction vector $\mu$ is calculated, and the similar method is used to estimate the motion parameters of the formula (6).

It is known that the equation (6) is ill conditioned, that is, its solution is not or is not unique. In order to reduce the ill condition, the formula (5) and (6) can be further modified to:

$$
\begin{aligned}
& \omega_{1}(F, \mu, t, \Delta t)=\left(\mu \cdot\left(\frac{\partial}{\partial x_{1}}, \frac{\partial}{\partial y_{1}}\right)+\frac{\partial}{\partial t}\right) K(\mathbf{v}, t) \\
& \omega_{2}\left(F, \frac{\mathrm{d} \mathbf{v}}{\mathrm{d} t}, t, \Delta t\right)=\left(\frac{\mathrm{d} \mathbf{v}}{\mathrm{d} t} \cdot\left(\frac{\partial}{\partial x_{1}}, \frac{\partial}{\partial y_{1}}\right)+\frac{\partial}{\partial t}\right) K(\mathbf{v}, t)
\end{aligned}
$$


In $K=f_{z}(F)$, where $f_{z}$ is a linear operation of the space integrable function, a convolution operation is used, whose kernel is a Gauss function. The formula (7) and (8) can be called the lattice Pohl Seidman equation and the auxiliary equation for the optical flow field of the linear operator $f_{z}$, and can solve the ill posed problem of the equation effectively.

\section{Lattice D2Q9 Model of Optical Flow Equation}

One of the most commonly used equations in dynamics is the time relaxed approach (single-relaxation-time SRT), which is the hatnagar - Gross - Krook (BGK) model [8]:

$$
\frac{\partial f}{\partial t}+\xi \cdot \nabla f=\frac{1}{\lambda}\left(f-f^{(0)}\right)
$$

Where $f^{(0)}$ is a balanced distribution function, that is the Maxwell Pohl Seidman (Maxwell-Boltzmann) distribution function, $\lambda$ is a slack time. The corresponding viscosity is $v=\lambda R T$, where $R$ is the gas constant, and $T$ is the temperature of the gas.

In order to get the solution of the function $f$, the finite velocity vector set $\left\{\xi_{\alpha}\right\}$ is used to discretize the formula (9) in the velocity space. The following equation can be obtained:

$$
\frac{\partial f}{\partial t}+\xi_{\alpha} \cdot \nabla f_{\alpha}=\frac{1}{\lambda}\left(f_{\alpha}-f_{\alpha}^{(e q)}\right)
$$

Where XXX is related to the first $\alpha$ discrete velocity $\xi_{\alpha}, f_{\alpha}^{(e q)}$ represents a balanced distribution function corresponding to the discrete velocity space. Nine speed square lattice model D2Q9 model, refers to the 2 dimensional 9 speed model.

The model has been widely applied to various two-dimensional flow models. In the D2Q9 model, the $e_{\lambda}$ is represented by the discrete velocity set:

$$
\left\{\begin{array}{l}
e_{0}=0 \\
\left.\left.e_{\alpha}=c(\cos (\alpha-1) \pi / 4), \sin (\alpha-1) \pi / 4\right)\right) \\
\quad \alpha=1,3,5,7 \\
\left.\left.e_{\alpha}=\sqrt{2} c(\cos (\alpha-1) \pi / 4), \sin (\alpha-1) \pi / 4\right)\right) \\
\quad \alpha=2,4,6,8
\end{array}\right.
$$

For the formula $c=\partial x / \partial t$, where $\partial x$ and $\partial t$ represent the lattice constant and the time step length. The equilibrium distribution function of the D2Q9 model can be expressed as follows:

$$
f_{\alpha}^{(e q)}=\rho w_{\alpha}\left[1+\frac{3}{c^{2}} e_{\alpha} \cdot u+\frac{9}{2 c^{4}}\left(e_{\alpha} \cdot u\right)^{2}-\frac{3}{2 c^{2}} u \cdot u\right]
$$

The $w_{\alpha}$ in the formula is a weighting factor:

$$
\left\{\begin{array}{l}
w_{\alpha}=4 / 9, \quad \alpha=0 \\
w_{\alpha}=1 / 9, \quad \alpha=1,3,5,7 \\
w_{\alpha}=1 / 36, \quad \alpha=2,4,6,8
\end{array}\right.
$$

Fluid density and fluid volume can be expressed as a discrete velocity space:

$$
\rho=\sum_{\alpha=0}^{8} f_{\alpha}=\sum_{\alpha=0}^{8} f_{\alpha}^{(e q)}
$$




$$
\rho u=\sum_{\alpha=1}^{8} e_{\alpha} f_{\alpha}=\sum_{\alpha=1}^{8} e_{\alpha} f_{\alpha}^{(e q)}
$$

In this model, the velocity of the fluid in the ideal state is: $c_{s}=c / \sqrt{3}, p=\rho c_{s}{ }^{2}$.

\section{Image Detection and Segmentation Based on Lattice Pohl Seidman Video}

In order to solve the formula (14) and (15), it is necessary to set the initial parameters in the equation, that is, a set of nonempty set of vectors, with the help of lattice Pohl Seidman's D2Q9 model. The set of nonzero vectors can be set up under the guidance of a priori, and then the optimal set can be optimized by iteration.

It is assumed that the initial nonempty vector set:

$$
E_{0}=\left\{e_{\alpha} \mid \alpha=0,1,2, \ldots ., 8\right\}
$$

For this equation to set the initial value of $e_{0}=(0,0)$, the use of the initial $E_{0}$ for the optical flow equation, the beginning is often not the most appropriate non null vector set. It is necessary to gradually approach the most suitable non empty prediction vector set by iteration method $[9,10]$.

For the pixel $P(x, y, t)$, according to the estimated vector set $E_{0}=\left\{e_{\alpha} \mid \alpha=0,1,2, \ldots ., 8\right\}$, the estimated speed is $K=d \mathbf{v} / d t$ and $\mathbf{v}=(x, y)$. Then the $E_{1}=\left\{K+e_{\alpha} \mid \alpha=0,1,2, \ldots ., 8\right\}$ can be calculated by iteration method, and other values can be deduced by the similar method:

$$
E_{0}, E_{1}, E_{2}, E_{3}, \ldots E_{i} \ldots, E_{n}
$$

Iterate the computer until it converges to less than the preset threshold.

$$
D(\mathbf{v}, t)=\|K\|^{2} \sum \gamma_{u}^{2}
$$

Here $\|K\|$ is the vector of the optical flow vector, $E_{n}$ represents the convergence vector set, $\gamma_{u}$ represents the vector characteristics of different regions. Through this operator, select the appropriate threshold for the $\mathrm{D}$, according to the $\gamma_{u}$ image can be divided into different motion vector area.

The algorithm is repeated several times to obtain $E_{0}, E_{1}, E_{2}, E_{3}, \ldots E_{i} \ldots, E_{n}$ until the final convergence results are obtained [11]. The general case can be assumed for any small positive $\varepsilon$, there is a positive integer $\mathrm{N}$, for any $m>N$ and $n>N$ can meet $\left|K_{m}-K_{n}\right|<\varepsilon$.

\section{Algorithm Experiment}

As shown in Figure 1 results of the algorithm simulation experiment on two video, left the original video, which represents the initial parameters in the above right under the guidance of prior $(\mathrm{N}=3$, $\varepsilon=0.5)$, right below the initial parameters $(\mathrm{N}=6, \varepsilon=0.1)$. The arrows in the figure represent the average velocity vectors of all pixels in the video frame image. The sub block size of the image segmentation in the experiment is $12 \times 12$. If the requirement of processing precision is not very strict, the speed of image processing is 10 times of the same size as the Laplasse filter. 


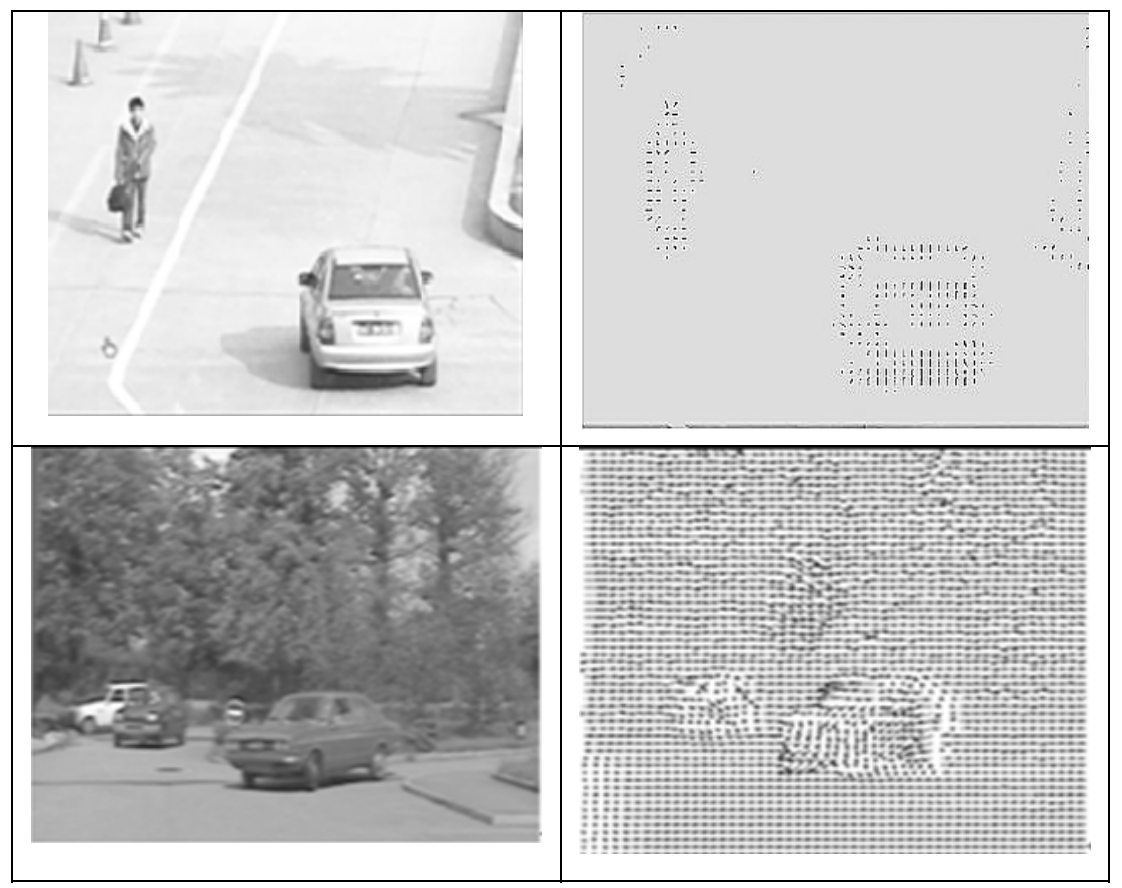

Figure 1 image segmentation and motion estimation using the proposed algorithm

\section{Summary and Prospect}

The content of this paper is to solve the problem of optical flow field by using the lattice Pohl Seidman method. The application of this method in the field of optical flow computation is presented. A formal lattice Pohl Seidman equation is established for the optical flow field in image sequences. The lattice D2Q9 model is used to segment the image by using the lattice Pohl Seidman equation. And the moving boundary can be detected by calculating the continuous motion vector field. The simulation results show that this method can accurately extract the moving object from the video image, the local motion detection accuracy, and compared to the traditional Laplasse filtering speed increased 10 times. This is of great significance for the real-time video coding and motion compensation.

\section{Reference}

[1] Dazhi Yua, Renwei Meia, Li-Shi Luob, Wei Shyya. Viscous flow computations with the method of lattice Boltzmann equation[J]. Progress in Aerospace Sciences. Volume 39, Issue 5, July 2013: 329-367.

[2] Rivet J-P, Boon JP. Lattice Gas Hydrodynamics[M] • Cambridge,UK: Cambridge University Press, 2011.

[3] Fletcher CAJ. Computational techniques for fluid dynamics[M] . vols. I and II. New York: Springer, 2013.

[4] B. Jawerth, P. Lin and E. Sinzinger. Lattice Boltzmann Models for Anisotropic Diffusion of Images[J]. Journal of Mathematical Imaging and Vision . Kluwer Academic Publishers, Netherlands, vol.11, no.3 2009::231-237.

[5] G. Aubert, P. Kornprobst. Mathematical Problems in Image Processing-Partial Differential Equations and the Calculus of Variations[M] . Springer-Verlag New York, LLC. 2012 :181-227. 
[6] C. Cofaru, W. Philips and W. Van Paepegem. Gradient- based Optical Flow for Sub-pixel Registration of Speckle Image Sequences Using a Spatial/Temporal Postprocessing Technique. IEEE ICIP 2008, San Diego, USA, October 2013:841-844.

[7] D.J. Fleet and Y. Weiss. Optical Flow Estimation in Mathematical models for Computer Vision. The Handbook, N. Paragios, Y. Chen, O.Faugeras (eds.), Springer 2015:239-258.

[8] Qian YH, d'Humières D, Lallemand P . Lattice BGK models for Navier Stokes equation[J] . Europhys Lett 2012. vol.17:479-484. .

[9] Q.S. Chang and T. Yang, A Lattice Boltzmann Method for Image Denoising - IEEE Transactions on Image Processing, vol. 18, no.12, 2009: 2797-2802.

[10] Y. Chen, Z.Z. Yan and J. Shi . Application of Lattice Boltzmann Method to Image Segmentation. in Proceedings of the 29th Annual International Conference of the IEEE EMBS Cite' Internationale, Lyon,France. August 2007:.6561-6564.

[11]Guang-Tai Ding, Shu-Qing Li, Dan-Xia Luo. Optical Flow Analysis Based on Lattice Boltzmann Method and Lower Order Approximation with Relaxation Factors . Multimedia Technology (ICMT), 2010 International Conference on. Oct. 2010:29-31. 